\title{
Fundamental issues and new trends in parallel mechanisms and manipulators
}

\author{
Clément Gosselin • Vincenzo Parenti-Castelli • \\ François Pierrot
}

Published online: 5 January 2011

(C) Springer Science+Business Media B.V. 2010

Parallel mechanisms and manipulators have been part of the robotics landscape for several decades. They have reached a certain level of maturity and commercial products based on these architectures are available for a variety of applications. Nevertheless, it is clear that the full potential of parallel mechanisms has not yet been exploited. Over the last two decades, numerous novel architectures have been proposed and new applications continue to emerge. In this context, research on parallel mechanisms and manipulators continues to flourish, creating a need for international forums in which researchers can exchange ideas and expose new results.

\section{Gosselin}

Département de Génie Mécanique, Université Laval, Pavillon Adrien-Pouliot, 1065 avenue de la Médecine, Québec, G1V 0A6, Canada

e-mail: gosselin@gmc.ulaval.ca

url: http://robot.gmc.ulaval.ca

\section{Parenti-Castelli ( $₫)$}

Department of Mechanical Engineering-DIEM, Università degli Studi di Bologna, Viale Risorgimento, 2, 40136 Bologna, Italy

e-mail: vincenzo.parenti@unibo.it

url: http://www.diem.ing.unibo.it/grab/

\section{F. Pierrot}

Laboratoire d'Informatique, de Robotique et de

Microelectronique de Montepellier (LIRMM),

161 rue Ada, 34392 Montpellier, France

e-mail: pierrot@lirmm.fr

url: http://www.lirmm.fr/ pierrot
This special issue of the journal features some of the papers that were presented at the Second International Workshop on Fundamental Issues and Future Research Directions for Parallel Mechanisms and Manipulators, held in Montpellier, France in September 2008. This workshop, organized by François Pierrot and his team at LIRMM, aimed at bringing together researchers and experts from around the world to discuss the state of the art and to identify promising future research directions for parallel mechanisms and manipulators. The workshop was a great success. Similarly to the first workshop held in Quebec City, Canada in 2002, the Montpellier workshop was not a routine conference but rather a focus of convergence where thorough presentations, focused paper sessions and interactive discussions provided a true scholarly atmosphere that led to fruitful open discussions.

Following the workshop, authors were invited to further improve their papers based on the discussions held at the workshop as well as on comments obtained from reviewers. The final result is presented in this issue. This collection of papers should be of great interest to the research community since it represents not only the isolated work of the authors but also the result of an interactive exercise that allowed the participants to nurture each other's thoughts. We are very grateful to the Meccanica team for the publication of this special issue which provides a high-quality archival opportunity for this work. 